\title{
Vulvar vitiligo-like depigmentation and multiple halos of hypomelanosis at the trunk following treatment with imiquimod $5 \%$ cream for vulvar condylomata: casual or related events?
}

\author{
Maíra Chiarelli Serra ${ }^{1,3}$ \\ Elisabetta Pennacchioli ${ }^{1,3}$
}

\author{
Claudia Menicanti ${ }^{2,3}$ \\ Giulio Tosti ${ }^{1,4}$
}

\section{DOI: http://dx.doi.org/10.1590/abd1806-4841.20143058}

\begin{abstract}
A 25-year-old Caucasian female with multiple genital warts involving the vulvar area was treated with imiquimod 5\% cream. During follow-up the patient developed areas of hypopigmentation at the site of application of imiquimod cream and areas of hypomelanosis around multiple preexisting nevi of the trunk. At 18 months follow-up genital depigmentation persisted and halo nevi of the trunk were still present. Different mechanisms of imiquimod-induced depigmentation have been reported. Halo nevi are considered expression of an autoimmune response. In the case presented here, it might be conceivable that both vitiligo-like depigmentation at the site of application and halo of hypomelanosis around melanocytic nevi have been induced by the same immunologic mechanism elicited by topical application of imiquimod.
\end{abstract}

Keywords: Condylomata acuminata; Immune system; Immune system phenomena; Nevus, halo; Vitiligo

\section{INTRODUCTION}

Imiquimod 5\% cream is currently approved for topical treatment of genital warts, superficial basal cell carcinoma and actinic keratoses. So far, several cases of vitiligo-like imiquimod-induced depigmentation have been reported. ${ }^{1,2}$ The present case report refers to the observation of vulvar depigmentation and onset of multiple halos of hypomelanosis around several melanocytic nevi of the trunk following treatment with imiquimod 5\% cream for vulvar condylomata (Figures 1 and 2). ${ }^{2}$

\section{CASE REPORT}

A 25-year-old Caucasian female with genital warts was treated with imiquimod 5\% cream three times weekly for 12 weeks. The treatment was well tolerated .At 4-month follow-up after discontinuation, the patient was noted to have some residual condylomata and marked depigmentation at the site of application.

At 8-month follow-up she was noted do have developed halos of hypomelanosis around four pre-

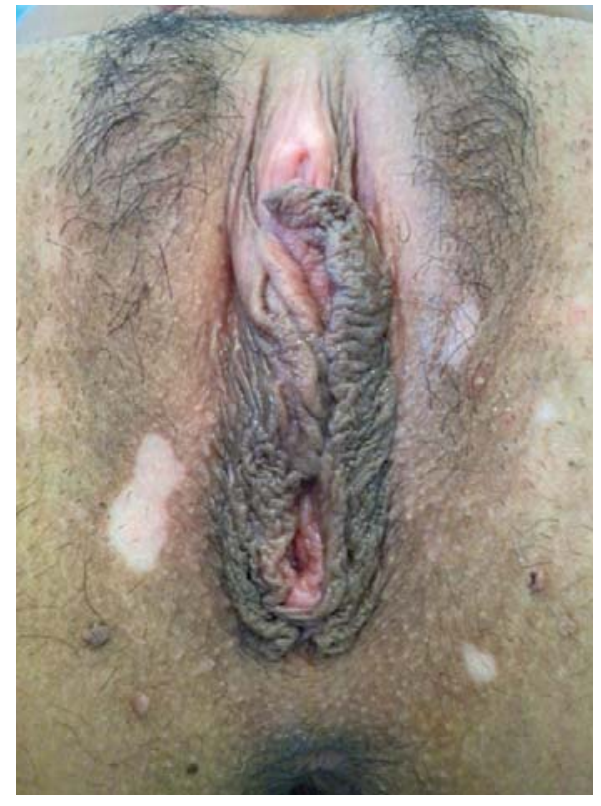

Figure 1: Multiple halos of depigmentation at site of Imiquimod application

\footnotetext{
Received on 16.08.2013.

Approved by the Advisory Board and accepted for publication on 20.09.2013.

Work performed at the Divisione Melanoma, Istituto Europeo di Oncologia - Milano, Italia.

Financial Support: None

Conflict of Interests: None.

Istituto Europeo di Oncologia -Divisione Melanoma- Milano, Italia.

Fondazione IRCSS Ca' Granda - Ospedale Maggiore Policlinico. Dipartimento di Fisiopatologia Medico-Chirurgica - Milano, Italia.

Università degli Studi di Milano- Milano, Italia.

Universita degli Studi di Ferrara - Ferrara, Italia.
} 


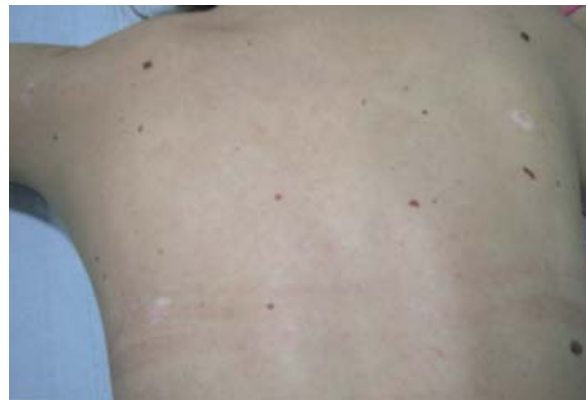

FigURE 2:

Several halos nevi (Sutton's nevi) of the trunk

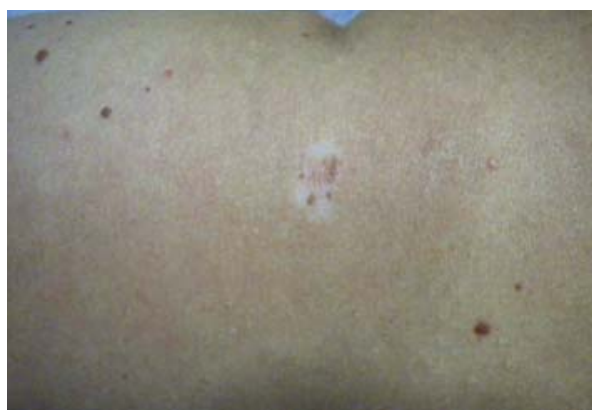

FIGURE 3:

Halo nevo in the left deltoid region

existing nevi of the trunk. At 18 months follow-up the genital depigmentation persisted and the halo nevi of the trunk were still present. Personal and family history for melanoma, vitiligo and thyroid disorders were negative .Laboratory investigations, including a panel of auto-antibodies, were performed without any significant abnormal finding.

\section{REFERENCES}

1. Speeckaert R, van Geel N, Vermaelen KV, Lambert J, Van Gele M, Speeckaert MM, et al. Immune reactions in benign and malignant melanocytic lesions: lessons from immunotherapy. Pigment Cell Melanoma Res. 2011;24:334-44.

2. Mashiah J, Brenner S. Possible mechanisms in the induction of vitiligo-like hypopigmentation by topical imiquimod. Clin Exp Dermatol. 2008;33:74-6.

3. Mendonça CO, Yates VM. Permanent facial hypopigmentation following treatment with imiquimod cream. Clin Exp Dermatol. 2006;31:721-2.

4. Stefanaki C, Nicolaidou E, Hadjivassiliou M, Antoniou C, Katsambas A. Imiquimodinduced vitiligo in a patient with genital warts. J Eur Acad Dermatol Venereol. 2006;20:755-6.

5. Gowda S, Tillman DK, Fitzpatrick JE, Gaspari AA, Goldenberg G. Imiquimod-induced vitiligo after treatment of nodular basal cell carcinoma. J Cutan Pathol. 2009;36:878-81.

6. Kim CH, Ahn JH, Kang SU, Hwang HS, Lee MH, Pyun JH, et al. Imiquimod induces apoptosis of human melanocytes. Arch Dermatol Res. 2010;302:301-6.

7. Ongenae K, Van Geel N, Naeyaert JM. Evidence for an autoimmune Pathogenesis of Vitiligo. Pigment Cell Res. 2003;16:90-100.

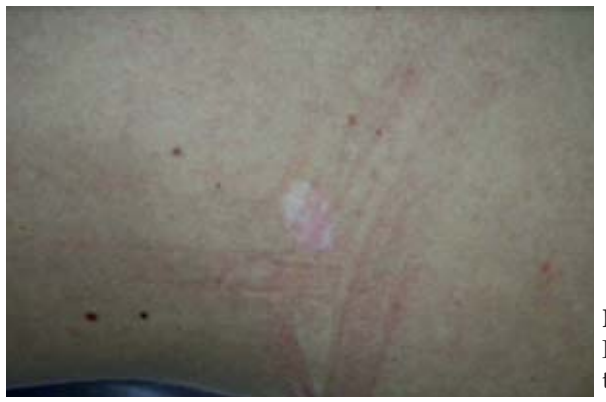

Figure 4:

Halo nevo of the trunk

\section{DISCUSSION}

The case presented here was characterized by the co-existence of genital vitiligo-like depigmentation and appearance of several halo nevi of the trunk. (Figures 3 and 4). ${ }^{3,4,5}$

Different mechanisms of imiquimod-induced depigmentation have been reported including postinflammatory depigmentation, necrosis of melanocytes and immune mediated vitiligo-like reactions. ${ }^{6,7}$

As vitiligo may be expression of a cytotoxic $\mathrm{T}$ lymphocyte immune reaction, it may be conceivable that imiquimod could contribute to the elimination of melanocytes by inducing acquired immunity through the induction of cytokines. ${ }^{1,6,}$

If this is true, it is likely that both vitiligo-like depigmentation at the site of application and halo of hypomelanosis around melanocytic nevi of the trunk have been induced by the same immunologic mechanism evoked by topical application of imiquimod. ${ }^{1,6,7}$

How to cite this article: Serra MC, Menicanti C, Pennacchioli E, Tosti G. Vulvar vitiligo-like depigmentation and multiple halos of hypomelanosis at the trunk following treatment with imiquimod $5 \%$ cream for vulvar condylomata :casual or related events? An Bras Dermatol. 2014;89(5)806-7. 